\title{
Successful Treatment with Clarithromycin for Rheumatoid Arthritis Accompanied by Infection
}

\author{
Masashi Ohe \\ Department of General Medicine, JCHO Hokkaido Hospital, Sapporo, Japan
}

\begin{abstract}
Macrolide antibiotics provide not only antibacterial activity but also anti-inflammatory effects. We report herein two cases of uncontrolled rheumatoid arthritis (RA), accompanied by hepatitis B and pulmonary empyema, respectively, who were successfully treated using clarithromycin (CAM) in expectation of its anti-inflammatory effects. A 74-year-old woman suffering from RA, presented with exacerbation of arthralgia. Because she was a hepatitis B carrier, she could not receive biological agents. She was successfully treated with CAM as an alternative to biological agents. A 77-year-old man suffering from RA, presented with exacerbation of arthralgia. Because he had suffered from pulmonary empyema, he did not receive biological agents. He was successfully treated with CAM as an alternative to biological agents. These present cases suggest that CAM treatment may be effective for RA.
\end{abstract}

Keywords: Rheumatoid arthritis; Clarithromycin; Anti-inflammatory agents

\section{INTRODUCTION}

Macrolide antibiotics (Macs) such as erythromycin, clarithromycin (CAM) and roxithromycin (RXM) provide not only antibacterial activity, but also anti-inflammatory effects. Regarding anti-inflammatory effects, successful treatments with Macs for diffuse panbronchiolitis, cystic fibrosis, chronic obstructive pulmonary disease, and bronchial asthma have been reported. Similarly, several recent studies have reported successful treatment of rheumatoid arthritis (RA) using Macs as anti-inflammatory agents [1-4]. We also report herein two cases of uncontrolled RA, accompanied by hepatitis B and pulmonary empyema, respectively, who were successfully treated using CAM in expectation of its anti-inflammatory effects.

\section{CASE REPORTS}

\section{Case 1}

A 74-year-old woman suffering from RA presented with exacerbation of arthralgia and/or articular swellings in bilateral shoulder, elbow, wrist, hand and knee joints. She had been diagnosed with
RA at 62 years old and had been administered salazosulfapyridine (SASP) at $1 \mathrm{~g} /$ day and subsequently methotraxate (MTX) at $6 \mathrm{mg} /$ wk in combination with loxoprofen (LOX) at $180 \mathrm{mg} /$ day. Her general condition, including arthralgia and articular swellings, had remained stable under this regimen for 10 years; however, arthralgia and articular swellings had worsened. We then investigated whether the patient was able to receive biological agents for RA. Positive results were obtained for hepatitis $B$ surface antigen, so she was deemed unable to do so. She was therefore treated largely successfully with prednisolone (PSL) at $5 \mathrm{mg}$ /day in combination with Celecoxib (CEL) at $400 \mathrm{mg} /$ day. Over 2 years after initiating PSL treatment, symptoms again started gradually deteriorating. On this visit, because 28 tender joint count (TJC28), 28 swollen joint count (SJC28), C-reactive protein (CRP) level and general health were 7, 3, $2.35 \mathrm{mg} / \mathrm{dL}$, and 70, respectively, disease activity score (DAS) 28CRP, defined as $0.56 \sqrt{T J C} 28+0.28 \sqrt{ } \mathrm{SJC} 28+0.36 \ln (\mathrm{CRP}[\mathrm{mg} / \mathrm{dL}] \times$ $10+1$ )+0.014 general health +0.96 (where general health is the patient assessment of disease activity using a $100-\mathrm{mm}$ visual analogue scale ranging from $0=$ best to $100=$ worst), was 5.21 (Table 1 ). Regarding disease activity, a DAS28-CRP over 5.1 indicates high disease activity, whereas a DAS28-CRP below 3.2 indicates low disease
Correspondence to: Masashi Ohe

Department of General Medicine, JCHO Hokkaido Hospital, 1-8-3-18 Nakanoshima, Toyohira-ku, Sapporo, Japan

Tel: +81-11-831-5151, Fax: +81-11-821-3851, E-mail: masshi@isis.ocn.ne.jp

Received: Jul. 18, 2015/ Accepted after revision: Aug. 13, 2015
(C) 2015 Soonchunhyang Medical Research Institute This is an Open Access article distributed under the terms of the Creative Commons Attribution Non-Commercial License (http://creativecommons.org/licenses/by-nc/3.0/). 
Table 1. Clinical data of case 1 before and after CAM treatment

\begin{tabular}{lcc}
\hline Variable & Before CAM treatment & After CAM treatment \\
\hline 28 Tender joint count & 7 & 4 \\
28 Swollen joint count & 3 & 1 \\
CRP (mg/dL) & 2.35 & 0.66 \\
General health & 70 & 30 \\
Disease activity score 28-CRP & 5.21 & 3.51 \\
\hline
\end{tabular}

CAM, clarithromycin; CRP, C-reactive protein.

Table 2. Clinical data of case 2 before and after CAM treatment

\begin{tabular}{lcc}
\hline Variable & Before CAM treatment & After CAM treatment \\
\hline 28 Tender joint count & 3 & 2 \\
28 Swollen joint count & 1 & 0 \\
CRP (mg/dL) & 0.99 & 0.30 \\
General health & 38 & 18 \\
Disease activity score 28-CRP & 3.59 & 2.52
\end{tabular}

CAM, clarithromycin; CRP, C-reactive protein.

activity. A DAS28-CRP between 5.1 and 3.2 indicates moderate disease activity [5]. Based on these criteria, her disease activity was classified as high. Because the PSL dosage could not be increased any further for fear of osteoporosis and hyperglycemia, CAM (400 mg/day) was added to PSL and CEL in expectation of anti-inflammatory effects on RA after obtaining informed consent. Two weeks after starting this treatment, arthralgia and articular swellings began to improve. Sixteen weeks after starting this treatment, arthralgia and articular swellings considerably improved. Because TJC28, SJC28, CRP level, and general health were 4, 1, $0.66 \mathrm{mg} / \mathrm{dL}$, and 30, respectively, DAS28-CRP was 3.51 (Table 1). Based on above-mentioned criteria, disease activity was considered moderate. CEL dosage could be reduced to $200 \mathrm{mg}$ /day.

\section{Case 2}

A 77-year-old man suffering from RA, which was largely controlled using MTX at $6 \mathrm{mg} / \mathrm{wk}$ in combination with prednisolone PSL at $5 \mathrm{mg}$ /day and CEL at $400 \mathrm{mg} /$ day, presented with gradual exacerbation of arthralgia and/or articular swellings in shoulder, wrist, and hand joint over 6 months. He had been diagnosed with RA at 72 years old. His arthralgia and articular swellings had remained stable under SASP (1 g/day) in combination with PSL (3 $\mathrm{mg} /$ day) and LOX (180 mg/day), and subsequently above-mentioned regimen for about 4.5 years. On this visit, because TJC28, SJC28, CRP level, and general health were 3, 1, $0.99 \mathrm{mg} / \mathrm{dL}$, and 38, respectively, DAS28-CRP was 3.59 (Table 2). Based on above-mentioned criteria, his disease activity was classified as moderate. MTX

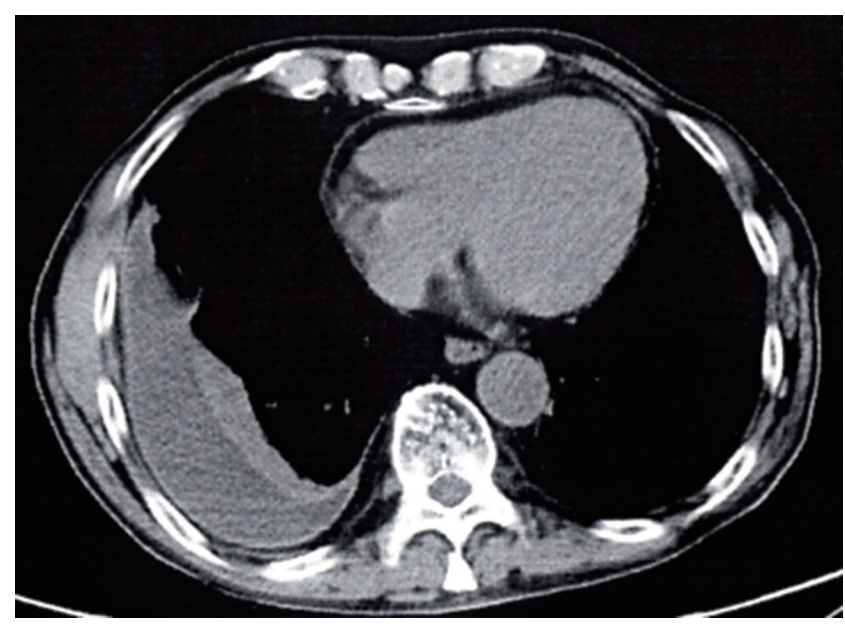

Fig. 1. Chest computed tomography showed right pleural effusion extending over the right chest wall.

dosage could not be increased any further because of leukocytopenia. Although he had suffered from controlled pulmonary empyema, the chest computed tomography (CT) findings of which showed right pleural effusion extending over the right chest wall (Fig. 1), he was recommended not to receive biological agents for fear of the exacerbation of pulmonary empyema. As an alternative, CAM at $400 \mathrm{mg} /$ day was therefore added in expectation of anti-inflammatory effects on RA after obtaining informed consent. Twelve weeks after starting this treatment, arthralgia and/or joint swellings improved considerably. Because TJC28, SJC28, CRP level, and general health were $2,0,0.30 \mathrm{mg} / \mathrm{dL}$, and 18 , respectively, DAS28-CRP was 2.52 (Table 2). Based on above-mentioned criteria, disease activity was considered low. CEL dosage could be reduced to $200 \mathrm{mg}$ /day. The pulmonary empyema shadow on chest CT remained unchanged after CAM treatment.

\section{DISCUSSION}

The introduction of biological agents such as tumor necrosis factor (TNF)- $\alpha$ blocking agents and an interleukin (IL)-6-receptor inhibitor has resulted in a paradigm shift in the treatment of RA [6]. Although these agents are efficacious against RA, serious adverse reactions such as fatal infections have been reported [7]. As a result, use of such agents is prohibited or not recommended for patients with current infections including hepatitis B or non-tuberculous mycobacterial infection. Because case 1 proved to be a hepatitis $B$ carrier, she was unable to receive these biological agents. As an alternative, she received PSL in combination with CEL. Because the 
Ohe M • Clarithromycin Treatment for Rheumatoid Arthritis

Table 3. European League Against Rheumatism response criteria

\begin{tabular}{llrc}
\hline \multirow{2}{*}{ Present DAS28 } & \multicolumn{3}{c}{ DAS28 improvement } \\
\cline { 2 - 4 } & \multicolumn{1}{c}{$>1.2$} & $>0.6$ and $\leq 1.2$ & \multicolumn{1}{c}{$\leq 0.6$} \\
\hline$\leq 3.2$ & Good response & Moderate response & No response \\
$>3.2$ and $\leq 5.1$ & Moderate response & Moderate response & No response \\
$>5.1$ & Moderate response & No response & No response \\
\hline
\end{tabular}

DAS, disease activity score

effectiveness of this treatment did not continue for the long-term, CAM was successfully added in expectation of its anti-inflammatory effects. Similarly, because case 2 had suffered from pulmonary empyema, he did not receive these biological agents. As an alternative, CAM was successfully added in expectation of its anti-inflammatory effects.

DAS28 can be used to assess disease activity status and treatment response properly and accurately [8]. According to European League Against Rheumatism response criteria (Table 3) [9], CAM treatment achieved moderate response in both cases.

As stated above, Macs provide not only antibacterial activity, but also anti-inflammatory effects. In terms of these anti-inflammatory effects, Macs have been shown to affect several pathways of the inflammatory process, such as the production of proinflammatory cytokines [2]. In fact, CAM has been shown to inhibit the productions of TNF- $\alpha$ and IL-6 [10], which are known to be associated with clinical features of RA. Macs also exert a substantial anti-inflammatory influence through their antibacterial activity. For instance, Macs are active against periodontopathic bacteria, which are powerful stimulators of TNF- $\alpha$ and other proinflammatory cytokines in humans [2]. Regarding CAM treatment for RA, Ogrendik [2] reported that treatment with CAM at $500 \mathrm{mg} /$ day for 6 months improved the signs and symptoms in patients with early active RA. Saviola et al. [1] reported that CAM (500 mg twice a day for the first 10 days, followed by $250 \mathrm{mg}$ twice a day for the long term) could be beneficial in RA patients who were not responsive to, or could not tolerate, disease-modifying antirheumatic drugs (DMARDs). They also reported the efficacy of adding CAM (500 mg twice per day for the first 15 days, followed by 500 mg/day for the remaining 15 days) to MTX and low-dose methylPSL in active RA [3]. Regarding RXM treatment for RA, Ogrendik [4] reported that treatment with RXM at $300 \mathrm{mg} /$ day for 3 months improved DAS28. In these reports, they speculated that the effectiveness of Macs against RA might be attributable to its anti-inflammatory effects and/or its anti-bacterial activity against oral anaerobic bacteria which was thought to play an important role in the pathogenesis of RA [1-4]. Based on these facts including our two cases, Mac treatment may be effective and particularly helpful for RA patients unable to receive biological agents, DMARDs, or increasing doses of PSL because of medical conditions. Compared with the previous reports, the present report comprised only two cases. At the same time, there were differences in Mac dosage and duration of prescription between the previous reports and the present report. Therefore, for the purpose of substantiating the findings of two cases, more researches to assess response rate and tolerability are required, before the present CAM treatment can be recommended on a wider basis.

\section{REFERENCES}

1. Saviola G, Abdi Ali L, Rossini P, Campostrini L, Coppini A, Gori M, et al. Clarithromycin in rheumatoid arthritis patients not responsive to disease-modifying antirheumatic drugs: an open, uncontrolled pilot study. Clin Exp Rheumatol 2002;20:373-8.

2. Ogrendik M. Effects of clarithromycin in patients with active rheumatoid arthritis. Curr Med Res Opin 2007;23:515-22.

3. Saviola G, Abdi-Ali L, Campostrini L, Sacco S, Baiardi P, Manfredi M, et al. Clarithromycin in rheumatoid arthritis: the addition to methotrexate and low-dose methylprednisolone induces a significant additive value: a 24-month single-blind pilot study. Rheumatol Int 2013;33:2833-8.

4. Ogrendik M. Efficacy of roxithromycin in adult patients with rheumatoid arthritis who had not received disease-modifying antirheumatic drugs: a 3-month, randomized, double-blind, placebo-controlled trial. Clin Ther 2009;31:1754-64.

5. Prevoo ML, van't Hof MA, Kuper HH, van Leeuwen MA, van de Putte LB, van Riel PL. Modified disease activity scores that include twenty-eightjoint counts: development and validation in a prospective longitudinal study of patients with rheumatoid arthritis. Arthritis Rheum 1995;38:44-8.

6. Kaneko Y, Takeuchi T. A paradigm shift in rheumatoid arthritis over the past decade. Intern Med 2014;53:1895-903.

7. Verhelst X, Orlent H, Colle I, Geerts A, De Vos M, Van Vlierberghe H. Subfulminant hepatitis B during treatment with adalimumab in a patient with rheumatoid arthritis and chronic hepatitis B. Eur J Gastroenterol Hepatol 2010;22:494-9.

8. Koo BS, Hong S, Kim YJ, Kim YG, Lee CK, Yoo B. Clinical characteristics of patients with rheumatoid arthritis who have sustained high erythrocyte sedimentation rates after clinical remission. J Rheum Dis 2014;21: 20-4.

9. Wells G, Becker JC, Teng J, Dougados M, Schiff M, Smolen J, et al. Validation of the 28-joint Disease Activity Score (DAS28) and European League Against Rheumatism response criteria based on C-reactive protein against disease progression in patients with rheumatoid arthritis, and comparison with the DAS28 based on erythrocyte sedimentation rate. Ann Rheum Dis 2009;68:954-60.

10. Tamaoki J, Kadota J, Takizawa H. Clinical implications of the immunomodulatory effects of macrolides. Am J Med 2004;117 Suppl 9A:5S-11S. 